\title{
Naturschutz und Naturgeschichte um 1900. Der Beitrag der Basler Vettern Sarasin
}

Christian Simon

\section{Summary}

Conservation and Natural History around 1900: The Contribution of the Sarasin Cousins

Some basic concepts for the creation of the Swiss National Park were derived from observations made in Sri Lanka, Indonesia and New Caledonia. European researchers feared that the study of «virgin nature» would no longer be possible, as various species would soon become extinct under the combined influences of colonial practices and profit-oriented capitalism. While the motives for protecting nature originated from experiences made in the southern hemisphere, their scientific concept of conservation was based on European natural history and the related theories of evolution. In the light of this approach, endangered zoological and botanical species as well as «primitive» varieties of man were appreciated as «documents» to be preserved within their original environment for future scientific reference and research. Museum collections and reservations (parks) were two types of repositories connected to each other by the same objective.

Keywords: Paul Sarasin (1857-1929), Fritz Sarasin (1859-1942), Carl Gottfried Semper (1832-1893), Ludwig Rütimeyer (1825-1895), natural history, history of nature conservation

Christian Simon, Universität Basel, Departement Geschichte, Hirschgässlein 21, 07934689 06, CH-4051 Basel, Schweiz (christian.simon@unibas.ch). 


\section{Zusammenfassung}

Naturschutz und Naturgeschichte um 1900: Der Beitrag der Basler Vettern Sarasin

Grundlegende Ansätze für die Schaffung des schweizerischen Nationalparks basierten auf Beobachtungen, die in Sri Lanka, Indonesien und Neukaledonien gemacht worden waren. Forschungsreisende aus Europa befürchteten, dass ein Studium «unberührter Natur» nicht länger möglich wäre, da verschiedene Arten wegen der kolonialen Erschließung und der profitorientierten, kapitalistischen Nutzung tropischer Ressourcen vom Aussterben bedroht seien. Während die Motive für die Bewahrung der Natur auf Erfahrungen beruhten, die in Südländern gemacht wurden, hatten die wissenschaftlichen Schutzkonzepte ihre Wurzeln in der europäischen Naturgeschichte und den damit verbundenen Entwicklungstheorien. In dieser Perspektive wurden bedrohte Tier- und Pflanzenarten ebenso wie «primitive» Varietäten des Menschen als «Dokumente» aufgefasst, die mit ihrer angestammten Umwelt als Belege für wissenschaftliche Arbeiten und Gegenstände künftiger Forschungen erhalten bleiben sollten. Sammlungen in Museen und Naturreservate bildeten zwei Typen von Schutzräumen, die untereinander durch dieselbe Zielsetzung verbunden waren.

\section{Einleitung}

Der Schweizerische Nationalpark feiert im Jahr 2014 sein hundertjähriges Bestehen. Zu diesem Anlass soll nachfolgend aufgezeigt werden, aufgrund welcher wissenschaftlichen Konzeptionen naturgeschichtlich orientierte Forscher aus Basel zu Beginn des 20. Jahrhunderts die Forderung nach einem nationalen Reservat vortrugen. Dementsprechend werden die Ergebnisse dieser Studie (Abschnitt 4) auf den Zusammenhang zwischen Biologiegeschichte und Naturschutz ausgerichtet. Dieser Zusammenhang ist bisher nur für den deutsch-schweizerischen Kontext bekannt, dem die Basler Akteure zuzurechnen waren; entsprechende Abklärungen für andere wissenschaftsgeschichtliche Felder bleiben ein Desiderat.

Der erste Teil (Abschnitt 1) ist einer Re-Historisierung der Biologiegeschichte gewidmet. Es wird dabei gezeigt, wie die Vorstellung einer Entwicklung der Arten (oft im Sinne einer Höherentwicklung) im Verlauf geologischer Zeiträume bereits vor dem Erscheinen von Charles Darwins Hauptwerk On the Origin of Species (1859) durch vergleichende Anatomie und Paläontologie zu einer wissenschaftlichen Gewissheit geworden war. 
Diese Vorstellung prägte die Rezeption von Darwins Evolutionslehre bei vielen seiner Zeitgenossen und führte zu einem Bias in der Schwerpunktsetzung der Darwin-Lektüre, indem «natural selection» zugunsten von Anpassung, Wanderungen und Isolierung in den Hintergrund geschoben wurde. Aus diesem wissenschaftsgeschichtlichen Kontext heraus entstanden die naturgeschichtlichen Ideen, die für eine spezifische Begründung des Naturschutzes um 1900 maßgebend wurden.

Im zweiten Teil (Abschnitt 2) wird die internationale Wissenschaftsgeschichte auf die lokalen Vorgänge in Basel bezogen. Der Beitrag eines weltweit vernetzten Erforschers der Entwicklungsgeschichte insbesondere in der Zoologie und Anthropologie, Ludwig Rütimeyer, wird dargelegt und gezeigt, wie zwei seiner Schüler, Paul und Fritz Sarasin, nach einer zusätzlichen Ausbildung bei Carl Semper in Würzburg als Forschungsreisende die Naturgeschichte von Sri Lanka, Sulawesi (Indonesien) und Neukaledonien erforschten. Die Erkenntnisse aus diesen Reisen ordneten sie in die von Rütimeyer übernommene und von der im ersten Teil diskutierten Darwinrezeption informierte Entwicklungskonzeption ein. Diese Konzeption wies drei Dimensionen auf: Die (taxonomische) Verwandtschaft zwischen Arten, die (geologische) Zeitachse ihrer Entwicklungsgeschichte und den (tiergeographischen) Raum der Verbreitung von Arten und Varietäten, einschließlich solchen des Menschen.

Der dritte Teil (Abschnitt 3) erläutert, inwiefern diese Entwicklungskonzeption eine Voraussetzung für den Naturschutzgedanken geworden ist. Individuen («specimina») von Arten und Varietäten wurden im Rahmen dieser Konzeption als «Dokumente» der Entwicklungsgeschichte «gelesen». Im Interesse der wissenschaftlichen Forschung mussten sie der Nachwelt in Repositorien erhalten bleiben. Dies geschah im 19. Jahrhundert bereits durch die Konservierung von Präparaten in Museumssammlungen; es sollten nun ergänzend Voraussetzungen zum Erhalt lebender und sich fortpflanzender «Dokumente» (Pflanzen, Tiere, Menschen) in ihren ursprünglichen, naturräumlichen und kulturellen Kontexten geschaffen werden. Neben ästhetischen Antrieben war somit die naturgeschichtliche Auffassung von Entwicklungsforschung ein wesentlicher Grund für die Forderung nach Reservaten. Dieser Begründungszusammenhang beruhte auf Einsichten, die in den Tropen gewonnen worden waren; sie wurden in der Zeit unmittelbar vor dem Ersten Weltkrieg einerseits auf Europa übertragen, andererseits in das Postulat eines «Weltnaturschutzes» eingebracht. 


\section{Entwicklung und Evolution - eine Rezeptionsgeschichte}

Die Geschichte der modernen Biologie wird üblicherweise auf die «moderne Synthese», bezogen, die als Schlüsselereignis dient. Die bekannteste Darstellung dieses Vorgangs stammt von Ernst Mayr (1904-2005), der selbst einer der «Architekten» dieser Synthese war. ${ }^{1}$

Es handelt sich dabei um die Geschichte einer Kombination aus verschiedenen Strängen der Biologie, nämlich der experimentellen Genetik der Drosophila von William Hunt Morgan («Lord of the Flies»²) und Hermann Joseph Mullers Experimenten zu Mutationen ${ }^{3}$ mit der mathematischen Populationsgenetik, für die der Name von Thedosius Dobzhansky steht, ${ }^{4}$ der Molekularbiologie von Physikern wie dem «Phagen-Delbrück» ${ }^{5}$ und den Biologen Crick und Watson, die die DNA-Strukturhypothese veröffentlicht haben. ${ }^{6}$ Die Zusammenführung der Elemente, die in der Wahrnehmung vieler Zeitgenossen sich eher gegenseitig ausschlossen als ergänzten, so namentlich im Verhältnis zwischen Darwins «natural selection» und der ursprünglichen Genetik, kann metaphorisch als Bildung eines «Hybrids» verstanden werden. Sie erstreckte sich gemäß dieser Erzählung über einen Zeitraum von mehreren Jahrzehnten und war damit eher ein Prozess als ein wirkliches Ereignis. Die Verbindung dieser Ansätze des 20. Jahrhunderts sollte gemäß der erwähnten historiographischen Konzeption die naturwissenschaftlich-empirische (molekularbiologische und statistisch-genetische) Erklärung nachliefern für die im Rahmen der Naturgeschichte des 19. Jahrhunderts von Charles Darwin (1809-1882) formulierten Lehre: Es gibt einen Prozess namens «Evolution»; dieser beruht auf einem Mechanismus namens «natural selection», der aus genetischer Variation, Isolierung, Selektion und Vererbung besteht. ${ }^{7}$

Historiker sind nicht nur Geschichtenerzähler, sie sind auch Spezialisten für die Dekonstruktion von Geschichten. Was für Forschende in den Naturwissenschaften die befriedigende geschichtliche Herleitung einer wissenschaftlichen Erkenntnis ist, die ihrem eigenen Tun Sinn verleihen hilft, ist für den Spezialisten der erzählten Geschichten eine Konstruktion aus verschiedenen Elementen, die untereinander durch Rhetorik verbunden sind. Die «moderne Synthese» ist in diesem Sinne ein Hybrid, und deren Erzählung

1 Mayr 194; Mayr/Provine 1980; Mayr 1982; Bowler 2009.

2 Kohler 1994.

3 Carlson 1981.

4 Adams 1994.

5 Fischer/Lipson 1988; Cairns/Stent/Watson 1992.

6 Chadarevian 2003.

7 Smocovitis 1996. 
bildet ein typisches «master narrative» oder «grand narrative». ${ }^{8}$ Was Darwin selbst unter Evolution verstanden hat, bleibt eine Frage der Forschung, die noch viele Generationen beschäftigen wird, denn sie ist nicht abschließbar. Wir können die Gedanken Darwins nicht denken, und könnten wir es, hätten wir nicht seine Sprache, um sie auszudrücken. Es gibt nur Annäherungen und Interpretationen - damit beschäftigt sich die «Darwin industry». ${ }^{9}$

Der wichtigste Weg historischer Annäherung ist die Kontextualisierung. In unserem Zusammenhang besteht die Aufgabe darin, die Texte Darwins mit anderen Texten aus seiner Zeit zu konfrontieren. Man sieht dadurch nicht schärfer, aber man kann verfestigte Voraussetzungen relativieren, Geschichten wieder offen machen, die von den Handbuchgeschichten «geschlossen» worden sind. Dadurch gewinnen sie neue Attraktivität. Ein anderer wichtiger Weg führt über das Studium von Rezeptionen: Was Gedanken in Wissenschaft und Gesellschaft bewirkten, hängt davon ab, was sich Leser und Zuhörer darunter vorgestellt haben, mit anderen Worten von deren Rezeption, weniger davon, was ein Autor wirklich gewollt hat. ${ }^{10}$

Die Arbeiten von Sander Gliboff sind in dieser Hinsicht lehrreich. ${ }^{11}$ Für die Wirkung Darwins unter der Mehrzahl seiner Zeitgenossen war nicht das Revolutionäre, Unerhörte und für die Ohren Wohldenkender auch Ungehörige des Gedankens der Evolution maßgebend, sondern sie versuchten, in Darwin eine Bestätigung des bereits Bekannten oder doch Vermuteten zu finden, dass nämlich die Arten des Tier- und Pflanzenreichs eine Geschichte hätten, und dass sie in dieser Geschichte untereinander in Verwandtschaft verbunden seien.

Charles Darwin erscheint in dieser Perspektive als der ideale Forschungsreisende und der vorbildliche, aus eigenen Mitteln lebende Gentleman, der nach seiner Reise auf der Beagle (1831-1836) das gesammelte Material und die Notizen ein Leben lang ordnete, verglich, auf andere Sammlungen bezog und damit zum wahren «Naturalisten» oder Naturgeschichtler wurde. Als solcher pflegte er sein weltumspannendes Wissen, tauschte sich mit seinesgleichen im Gespräch oder durch den Briefwechsel aus und verfasste gelegentlich eine Publikation, mit der er weitere Kreise an seinem immensen Schatz von Erfahrung teilhaben ließ. Nach dem Zeugnis vieler Darwinleser des 19. Jahrhunderts verschwand das für uns Revolutionäre fast völlig hinter diesem Bild. Denn was durch diese Art der Lektüre aus dem Blickfeld ausgeschieden wurde, war fast immer der Mechanismus der Auswahl («natural

8 White 1973; Lyotard 1979.

9 Lenoir 1987.

10 Warning 1975.

11 Gliboff 2005. 
selection»), der Faktor der fehlenden Richtung der «natural selection», des «Zufalls» oder genauer der «Zielfreiheit» der Evolution.

Sander Gliboff hat am Beispiel der deutschen Darwin-Rezeption gezeigt, dass gerade Darwins Anhänger diese Art der Rezeption pflegten. ${ }^{12}$ Die deutsche Rezeption ist hier einschlägig, weil nachher die Beziehungen zwischen Naturgeschichte und Naturschutz anhand einer Basler Geschichte, derjenigen von Paul und Fritz Sarasin, behandelt werden wird, die ihre wissenschaftliche Weiterbildung in Deutschland bezogen hatten.

Heinrich Georg Bronn (1800-1862) war der erste deutsche Darwin-Übersetzer. ${ }^{13}$ Bronn, selbst einer der damals führenden Paläontologen, hatte seit den 1820er Jahren nach Gesetzen, die die «Entwicklung» leiten, gesucht. «Entwicklung» war für ihn eine Anpassung des Körpers und dessen Baus an äußere Umstände. In seiner Übersetzung kritisierte er Darwin für die zentrale Bedeutung, die der britische Forscher der «natural selection» zusprach, denn diese wurde als anonyme Kraft eingeführt. Bronn vermutete dahinter Metaphysik statt Wissenschaft, d.h. ein säkularisiertes «Schöpfer»-Konzept. Auch für die Analogie zwischen der natürlichen Selektion und der Züchtung von Haustieren durch den Menschen, die für Darwin ein wichtiges Moment in seinem Argument war, hatte Bronn kein Verständnis. «Entwicklung» identifizierte der deutsche Übersetzer wie die meisten Morphologen mit «Vervollkommnung»..$^{14}$

Obschon der in Würzburg lehrende Carl Semper (1832-1893) heute nicht zu den prominentesten Darwinisten gerechnet wird, war er in seiner Epoche einer der bekanntesten Naturgeschichtler. ${ }^{15}$ Semper hatte sich von 1858 bis 1865 auf den Philippinen aufgehalten, ${ }^{16}$ er war wie Bronn, aber auch wie der Doyen der deutschen Entwicklungsforschung, Karl Ernst von Baer (1792-1876), ${ }^{17}$ schon davon überzeugt gewesen, dass Arten eine Geschichte hätten, bevor Darwins Hauptwerk 1859 publiziert wurde. Was Semper und andere in Darwin fanden und was sie begeisterte, war die mit überzeugender, reicher Gelehrsamkeit vorgetragene Beweisführung durch Indizien, dass die Arten sich in der Geschichte veränderten. «Survival of the fittest» war ein Ansatz, den zum Beispiel Semper lebhaft bejahte. Die Selektion hingegen erregte keine besondere Neugier; auf dieser Vorstellung wurden keine

12 Gliboff 2008.

13 Nyhart 1995, 106ff.; Gliboff 2007.

14 Gümbel 1876; Gliboff 2008.

15 Mayr 1975. Biographische Notiz zu «Karl Gottfried Semper» in: Jahn 2000, 954 (die Schreibweise des Vornamens schwankt zwischen Karl und Carl; in den Basler Dokumenten wird immer Carl geschrieben).

16 Semper 1867-1906.

17 Riha/Schmuck 2011; Baer 1828/1837. 
Programme aufgebaut. Denn da schon vor der Bekanntschaft mit Darwins «Origin» vorausgesetzt wurde, dass Arten einem Wandel unterworfen waren, folgte aus dem Wissen über die Tatsache des Artenwandels das Programm einer Erforschung der Anpassung von Formen des Lebendigen an äußere Gegebenheiten. Die Grundprinzipien Darwins lauteten in dieser Lesart, die Carl Semper praktizierte, dass die Organismen über Anpassungsfähigkeit verfügten (durch «Umbildung») und dass sie erworbene Eigenschaften zu vererben vermochten. Mutationen galten nicht als entscheidend, und Selektion war nach dieser Auffassung ein zielgerichteter Prozess («Entwicklung»). Der Morphologie wurde die Aufgabe zugedacht, zwischen vererbten Eigenschaften und kürzlich entstandenen Anpassungen zu unterscheiden.

Darwin, so meinte Semper, habe zugegeben, dass «er sowohl den umbildenden als auch den auswählenden Einfluss der äußeren Existenzbedingungen unterschätzt habe». Nur aus rhetorischen Gründen habe er den auswählenden Einfluss («natural selection») zu Lasten der Anpassung in den Vordergrund gestellt. ${ }^{18}$ Semper entwickelte daraus das Programm seiner Untersuchungen über den Einfluss der Umwelt auf die Tierwelt - Thema seines Hauptwerks «Die natürlichen Existenzbedingungen der Tiere», das auf einer Reihe von in Boston abgehaltenen Vorträgen beruhte und internationale Anerkennung fand. ${ }^{19}$ In dieser Hinsicht unterschied er sich nur geringfügig von Ernst Haeckel (1834-1919), den er ansonsten hasste..$^{20}$ Interessant für das 19. Jahrhundert war somit nicht die Frage nach dem Mechanismus der «natural selection» (wie in der modernen Darwinlektüre), sondern die Frage nach der Entstehung der Arten - woher kommt das Neue in die Vielfalt? Das Ergebnis des vollzogenen Anpassungsvorgangs wurde nach dieser Auffassung (unter den selektionierten Individuen) vererbt, wobei in der Embryonalentwicklung eine Art Rekapitulation dieses Prozesses erkannt wurde. Niemand dachte in Populationen, man argumentierte nur mit Individuen. ${ }^{21}$

Den Verlauf des Artenwandels stellte man sich chronologisch rückwärts wie einen Stammbaum vor. So viel fand man bei Darwin bestätigt, dass Arten untereinander verwandt seien und dass die rezenten Arten von älteren, «primitiveren» Arten abstammten («Deszendenz»)..$^{22}$ Demgegenüber kann

18 Semper 1880, 1. Teil 4, 44; vgl. Junker 2000, 382-384.

19 Semper 1879. Das Hauptwerk ist in unserer Auffassung Semper 1880; vgl. Nyhart 1995, 177f., $308 \mathrm{ff}$.

20 Manche Forscher lehnten Ernst Haeckel nicht nur deshalb ab, weil er aus Naturwissenschaft eine Weltanschauung ableitete, sondern auch darum, weil er als Betrüger galt. Diese Auffassung beruhte auf einer Rezension durch den Basler vergleichenden Anatomen Ludwig Rütimeyer (Richards 2005,17).

21 Haeckel 1866. Biographische Notiz zu «Ernst Haeckel» in: Jahn 2000, 840f.; Junker 2000, 356.

22 Schmitt 2010, 3-5 zu den Begriffen Entwicklung, Deszendenz und Abstammung. 
die neuere Darwinforschung plausibel machen, dass Darwin selbst gerade nicht an einen «Baum» dachte, und schon gar nicht an die Entwicklung vom Einfacheren zum Höheren, Komplexeren. Für die Leser der 1860er und 1870er Jahre galt jedoch das kleine Schema in Darwins «Origin» als eine Baum-Metapher, die für sie wegweisend wurde..$^{23}$

Insofern als der Mensch mit seinem Leib ein Objekt der vergleichenden Anatomie und der Zoologie darstellte, musste er im Einklang mit der Vorstellung der Deszendenz selbst auch einen Stammbaum haben, der ins Tierreich hinabreichte. Für die teilsäkularisierte Naturwissenschaft der 1860er Jahre und danach war es kein Skandalon, in diesem Stammbaum Affen zu sehen. ${ }^{24}$ Carl Semper meinte 1876, Darwin zeige, dass das Tierreich eine in sich verwandte Familie sei. Neu bei Darwin sei die Idee eines «materiellen Zusammenhangs», eines «großen erklärenden Gesetzes», wobei Darwin den Menschen «mitten in die Thierwelt hinein» stelle. Da die Zoologie den Naturwissenschaften zugehöre, dürfe sie wie z.B. die Chemie nicht nach der Würde des Menschen fragen. Ihre Aufgabe sei vielmehr, die «materiellen Erscheinungen nach dem Gesetz des Causalzusammenhangs» zu erklären. Transzendentale Spekulationen hätten in den Naturwissenschaften keinen Platz; als Wissenschaften suchten sie bloß Wahrheit. ${ }^{25}$

Der «Stammbaum» der Arten wurde auch vorwärts gelesen, nämlich als Entwicklungsplan - und hier wich die Rezeption deutlicher von Darwin selbst ab, als dies in der Geringschätzung der Selektionsthematik der Fall war. Diese beiden Akzentsetzungen hingen miteinander zusammen, denn wer Entwicklung als planmäßigen Prozess verstand, musste sich um Selektionsmechanismen weniger kümmern als um die schrittweise Anpassung der Baupläne der Tiere an deren Umwelten und um die fortschreitende Höherentwicklung. ${ }^{26}$

«Reine Wissenschaft» war ein Ideal, das in der ersten Hälfte des 19. Jahrhunderts mit dem Ziel etabliert worden war, das Naturwissen von christlichen Lehren und naturphilosophischen Annahmen frei zu halten. Glaube und Weltanschauung sollten den Fortschritt in der Naturerkenntnis nicht durch Einmischung behindern, aber Wissenschaft sollte den Wissenschaftlern vorbehalten bleiben, die sich demzufolge hüteten, öffentlich Schlussfolgerungen aus ihren Erkenntnissen für Weltanschauung und Religion zu ziehen. Ebenso wichtig war die Distanzierung von einer Wissenschaftsauf-

23 Sarasin, Ph. 2009, 187, 189, 241 zu Darwins Intention und den Missverständnissen in den Rezeptionen.

24 Darwin 1871.

25 Semper 1876.

26 Shanahan 2004. 
fassung, die die Deduktion aus allgemeinen philosophischen Prinzipien zuließ - das Newtonsche «hypotheses non fingo» galt als Grundlage seriöser, d.h. empirischer Forschung. ${ }^{27}$

Neben einem solchen Darwinismus, der sich als rein wissenschaftlich verstand, gab es Weltanschauungen, die sich auf Darwin beriefen. Zu den kulturpolitischen Darwinisten rechnen wir diejenigen Autoren, die Darwins Lehre als Beitrag zur Aufklärung und Emanzipation des Bürgers von Legenden, Kirche und Klerus verwendeten, bis hin zu den Monisten, die die Scheidung Geist-Körper aufgaben, und solchen, die auf materialistischer Grundlage Darwin rezipierten. Dem kulturpolitischen Darwinismus ging es um die aufklärerisch-rationale Befreiung des Menschen von Aberglauben und von nicht-rational fundierter Autorität. Die Abstammungslehre wurde als Antithese zur Schöpfungslehre aufgezogen, der Darwinismus als Religionsersatz des rationalen, säkularisierten, wissenschaftlich gebildeten Bürgers verwendet. ${ }^{28}$ Nachdem die Republikaner die politische Herrschaft in Frankreich erlangt hatten, stifteten sie als wissenschaftsgläubige Aufklärer an der Sorbonne 1888 einen Lehrauftrag für Entwicklungslehre («Evolution des êtres organisées»), den der radikale Député von Valenciennes, Alfred Giard (1846-1908), erhielt. ${ }^{29}$ In Deutschland popularisierte Ernst Haeckel eine Darwin-Lesart als neue Weltanschauung. «Reinen» Wissenschaftlern wie Semper war dies ein Gräuel. ${ }^{30}$

\section{Nach Basel und in die Welt hinaus}

Nicht ohne Absicht haben wir Semper erwähnt. Paul und Fritz Sarasin (18571929 resp. 1859-1942), zwei junge Basler, doktorierten bei ihm im Abstand von wenigen Jahren. ${ }^{31}$ Die beiden waren als «Großvettern» entfernt miteinander verwandt und gehörten der finanziellen und politischen Elite des Basler Stadtstaates an. Fritz entstammte der Familie des Bürgermeisters und Baumwollindustriellen Felix Sarasin, Paul war ein Sohn des Ratsherrn

27 Exemplarisch ist die Ablehnung «reiner Hypothesen» durch Semper 1880, 2. Teil, 253. Anregend Green 2010, 698-700.

28 Arnst/Jaeschke 2000; Gregory 1977.

29 Bouyssi 2001; Loison 2010, $24 \mathrm{ff}$.

30 Bayertz/Jaeschke/Gerhard Band 1, 2007; Wuketits 1998; Richards 2008; Weingart/Kroll/ Bayertz 1992; Weingart 1994; Semper 1876.

31 Für Informationen über Paul und Fritz Sarasin ist man auf die Nekrologe und einen Begleitband zu einer Ausstellung angewiesen: Sarasin, F. 1928/29; Stehlin 1930; Rütimeyer, Leopold 1931; Speiser 1943; Gedächtnis-Ausstellung 1959. Zur Einordnung in die Stammtafel der Familie Sarasin siehe www.stroux.org, besucht 11.6.2012. 
und Seidenbandunternehmers Karl Sarasin. ${ }^{32}$ Beide arbeiteten nie um Lohn, sondern wirkten, gestützt auf eigenes Vermögen, nur für die Wissenschaft und die Vaterstadt, was dem doppelten Ideal der «reinen Wissenschaft» der «Gentlemen scientists» und dem des Patriotismus entsprach.

Wie damals in Basel üblich, hatte Paul die ersten Semester an der heimischen Universität verbracht, während Fritz sein Studium in Genf begonnen hatte, bevor er Paul bei den Anlässen der Studentenverbindung «Zofingia» an der Basler Universität traf. Da Basel noch in den 1880er Jahren in den Naturwissenschaften (namentlich in Zoologie und Botanik) vor allem Propädeutik anbot, nutzten die meisten an Wissenschaft und Forschung wahrhaft interessierten jungen Männer die Basler Universität für eine Vorbereitungsphase und wanderten dann an wichtigere Universitäten $a b$.

Dennoch lehrten hier bedeutende Persönlichkeiten. Im Medizinpropädeutikum war dies vor allen der vergleichende Anatom und Zoologe Ludwig Rütimeyer (1825-1895), ein Schüler des Berner Geologen Bernhard Studer (1794-1887). Der Pfarrersohn Rütimeyer hatte nach wissenschaftlichen Wanderjahren in Paris, Südfrankreich, London und Italien in Basel einen Lehrstuhl erhalten, den er von 1855 bis 1895 versah. In den letzten Lebensjahren widmete er sich zunehmend dem naturhistorischen Museum, das er - in Abgrenzung von der vor allem lehrenden Universität - als Hochburg der reinen Naturerkenntnis durch Forschung an Objekten verstand. ${ }^{33}$

Rütimeyer war trotz seiner etwas gesuchten, an Gotthelf gebildeten Schreib- und Sprechweise ein Gelehrter von internationalem Ansehen mit einem Netzwerk, das ihm Zugang zum aktuellsten Wissen über die Naturdinge eröffnete. Die Überzeugung, dass Arten eine Geschichte hätten, die sich durch das Studium von Fossilien, von Grabungsfunden und durch den Vergleich der Skelette rezenter Arten aus der ganzen Welt erforschen ließe, festigte sich bei Rütimeyer, als er die Knochenfunde aus Pfahlbauten und ehemals bewohnten Höhlen der Schweiz bearbeitete. ${ }^{34}$ Die Knochenkunde (Osteologie) führte Darwins Korrespondenzpartner ${ }^{35}$ zum Aufbau einer umfassenden Sammlung für das vergleichende Studium des Skelettbaus von

32 Pauls Vater Karl Sarasin (1815-1886) war der führende konservativ-christliche Politiker der Stadt. Janner 2012, 265-268, u.ö. Paul verarbeitete seine Erfahrungen auch in literarischen Texten, die ein zwar elitär-konservatives, aber doch gezielt orthodoxiefeindliches Programm zum Ausdruck brachten, z.B. Sarasin, P. 1893; Sarasin, P. 1908. Andere Arbeiten dieser Art erschienen unter dem Pseudonym F. Wiegand, z.B. Wiegand 1900.

33 Rütimeyer, Ludwig 1898; Sarasin, P. 1900; Meyer von Knonau 1907; His 1941,202-212; Balmer HLS online. Eine aktuelle wissenschaftsgeschichtliche Untersuchung über Rütimeyer existiert nicht.

34 Rütimeyer, Ludwig 1875.

35 Darwin Correspondence Database, http://www.darwinproject.ac.uk/namedef-3905, besucht 11.6.2012. Der Database sind 7 Briefe bekannt (1861-1874). 
Säugetieren (vor allem Haustieren) und Schildkröten. In seinen Aufsätzen und publizierten Ansprachen (die Vorlesungen haben keine Spuren hinterlassen) finden sich zwar verklausulierte, aber einigermaßen verständliche Aussagen über die Geschichte der Arten über geologische Zeiten hinweg, deren Verwandtschaft und die Rolle von Sammlungen zur Klärung dieser Verhältnisse. ${ }^{36}$

In dieser Hinsicht sah er sich durch Darwin in seinen eigenen Erkenntnissen zwar grundsätzlich bestätigt. Rütimeyers Anthropologie unterschied sich aber deutlich von derjenigen Darwins und stand den Ansichten der Naturgeschichtler des früheren 19. Jahrhunderts näher, die im Menschen wenn nicht die Krone der Schöpfung, so doch den Zielpunkt der Entwicklung sahen. Denn nur oder erst der Mensch verfüge über den Geist, der es ihm gestatte, sich über das verwesliche Fleisch zu erheben und nach Gott zu fragen. Zwei Arten von Gesetzen, die äußeren mechanischen, und die inneren, die sich gegen die von äußeren Kräften auferlegten Regeln sträubten, beherrschten sein Dasein. Das Sterbliche sei der Gesetzmäßigkeit unterworfen, aber es gebe Ziele, denen alles Leben zustrebe, und für diese gälten solche Gesetze nicht. Der gläubige Christ Rütimeyer wollte vermeiden, in der Basler Öffentlichkeit als Verächter der biblischen Lehren missverstanden zu werden, da er wie andere nach ihm deren Differenzierungsvermögen als gering, die Bereitschaft zur Verurteilung aber als groß einschätzte. Wichtig war ihm, wie später seinen Schülern, den Großvettern Sarasin, die Freiheit der Wissenschaft von weltanschaulicher und religiöser Einmischung, aber nicht weil die Wissenschaft die Religion ersetzen könnte, sondern weil sie ein eigenes geistiges Reich bilde. Wissenschaftliches Wissen war für Rütimeyer mit dem Gottesglauben vereinbar, wenn auch nicht mit einem wörtlichen Bibelglauben; er verstand es als Sieg des Geistes über die Materie. ${ }^{37}$ Naturgeschichte war somit keineswegs ein Weg zum Materialismus, auch wenn bedeutende Forscher wie zum Beispiel Carl Vogt (1817$1895)^{38}$ materialistische Neigungen zeigten. Der Basler vergleichende Anatom wusste diesen Kollegen fachlich trotzdem zu schätzen.

Aufschlussreich sind die Betrachtungen, die Rütimeyer anlässlich von Charles Darwins Tod anstellte. Dessen Suche nach einer Erklärung der

36 Rütimeyer, Ludwig 1865, 1868, 1876.

37 Rütimeyer, Ludwig 1865. Ein Einfluss christlicher Einstellungen auf die Naturforschung in Basel ist auch noch für spätere Generationen zu berücksichtigen, vgl. die Auseinandersetzung zwischen Paul Sarasin und Hermann Christ-Socin (1833-1933, vgl. Nekrolog in Verhandlungen der Schweizerischen Naturforschenden Gesellschaft 1934,493-511, und Lienhard, «Christ, Hermann») in: Staatsarchiv Basel-Stadt, Privatarchiv 212a, T2 XXIII Nr. 18-23a, insbesondere Schreiben vom 9.1.1909, 16.1.1909 und 6.2.1909. Portmann 1965.

38 Carl Vogt musste 1849 Gießen aus politischen Gründen verlassen, vgl. Geus 2000, $335 \mathrm{f}$. Biographische Notiz «Karl Christoph Vogt» in: Jahn 2000, 982. 
Geschichte der Arten außerhalb des Gedankens einer gerichteten und letztlich gottgewollten Entwicklung lehnte er ab. Noch mehr kritisierte er die kulturpolitische Verwendung von Darwins Ideen durch Ernst Haeckel, und er tendierte dazu, den Entwicklungsansatz bei Darwin als zwar gut durchgearbeitet und vor allem als empirisch sorgfältig untermauert, aber nicht als eigentlich radikal neu dazustellen. Er unterschied zwischen dem Naturgeschichtler Darwin, den er unter die hervorragendsten Entwicklungsforscher und Forschungsreisenden einordnete, und einem schlechten Anthropologen Darwin, dessen Lehren durch einen Rückgriff auf die Anerkennung der geistigen, transzendentalen Sphäre korrigiert werden müssten. Als Korrektiv empfahl er die Lektüre der Schriften von Karl Ernst von Baer: Die «Zielstrebigkeit» in der Natur, die diesem wichtig gewesen sei, erweise sich als «notwendiges Complement und vielleicht als Hintergrund zu der Darwin'schen Denkungsweise». Darwin vergesse die von Aristoteles und Kant geforderte Einstellung, «dass der Mensch vom Menschen so hoch als möglich denken solle», stellte Rütimeyer fest, aber er habe die von der Pietät gesetzten Grenzen nie überschritten - im Unterschied zu den Darwinisten. ${ }^{39}$ In seiner Rektoratsrede von 1867 hatte Rütimeyer Darwin noch ohne gröBere Kautelen als einen bedeutenden Forscher dargestellt, der das Richtige gesehen habe und dank dessen Türöffnung wir jeden Tag mehr über den Wandel der Arten lernten. Doch in «Die Grenzen der Tierwelt. Betrachtung zu Darwins Lehre» von 1868 insistierte er auf den Defiziten in Darwins Lehre: Der große Forscher tauge nicht zum Führer in der Metaphysik.

Paul und Fritz Sarasin betrachtete Rütimeyer als seine Schüler. Er steuerte deren Bildungsgang und wies ihnen den Weg nach Würzburg zu Carl Semper als Mentor im Rahmen einer eigentlichen «Fürstenerziehung». Darin vermittelte er ihnen nicht nur die Werte der reinen Wissenschaft als Leitlinien, sondern auch einen patriotischen Sinn, nämlich die Bereitschaft, die erworbenen wissenschaftlichen Kompetenzen letztlich der Vaterstadt zur Verfügung zu stellen. ${ }^{40}$

Was die Großvettern Sarasin von Rütimeyer zunächst lernten, war offensichtlich die Bedeutung von «Entwicklung» in einer Kontinuität von Buffon

39 Rütimeyer, Ludwig 1898, 381, 384f.

40 Die normative Vorstellung, dass Basel eine Stadtrepublik gewesen sei oder sein sollte, die auf Mäzenatentum und Engagement der Söhne der Stadt in konservativem Sinne angewiesen sei, erläutert Janner 2012, 177 u.ö. Nach 1875 bedeutete dieser patriotische Imperativ den Willen zur Erhaltung der Hegemonie der betreffenden Familien wenigstens in den kirchlichen, sozialen, kulturellen und wissenschaftlichen Bereichen. Überholt erscheinen auf dem Bielefelder Bürgertumsbegriff aufbauende Arbeiten wie Bachmann 1999. Informativ bleibt Burckhardt 1984. - Aufschlussreich für das Verhältnis zwischen Rütimeyer und den Vettern Sarasin ist seine «Rede bei der Sarasinfeier», 4. Juli 1890, abgedruckt in Rütimeyer, Ludwig 1902, 342-345. 
bis Darwin. Den praktischen Stadtpatriotismus akzeptierten sie hingegen erst nach langen Lehr- und Wanderjahren im Ausland. Rütimeyer war begeistert von den praktischen Studien der beiden jungen Naturforscher, zuerst von deren Arbeiten im Labor von Semper. Denn Naturgeschichte und Laborforschung bildeten keine Gegensätze, da die Embryologie, allgemeiner das Studium der Entwicklungsstadien der Tiere als Schlüssel zum Verständnis der Entwicklung aufgefasst wurde, neben der Paläontologie und neben der vergleichenden Anatomie. ${ }^{41}$ Danach freute sich Rütimeyer an den Erträgen der von den Sarasin selbst organisierten und finanzierten Reisen nach Ceylon. Dabei wurde ein Teil der Sammlungsobjekte sowie Belegstücke zu Publikationen jeweils ins Basler Museum geliefert, dort bestimmt und eingeordnet. Die Feldforschung, die Laborarbeit und das Museum als Repositorium und Stätte des Bestimmens und Einordnens ergänzten sich zu Orten einer Wissenschaft von der Entwicklung der Arten. ${ }^{42}$

Dabei gehörte «Entwicklung» nicht bloß in die zwei Dimensionen der (geologischen) Zeit und der Verwandtschaftsrelationen, die der Stammbaum symbolisierte. Sie hatte auch eine dritte, räumliche Dimension. Denn Entwicklung hieß auch Verbreitung, Zuwanderung,Verschiebung des Spektrums nachweisbarer Arten in geographischen Arealen (Tier- resp. Pflanzengeographie). Damit verband sie sich nochmals mit der Geschichte der Erde, nicht bloß paläontologisch, sondern historisch-geologisch. Den Untersuchungsraum hatten Forscher vor den Sarasin gewiesen, darunter Darwin selbst, aber vor allem Alfred Russel Wallace (1823-1913), der gegen Entgelt sammelte wie viele andere (auch der junge Rütimeyer) und deshalb kein GentlemanForscher war. Er hatte die «Wallace-Linie» postuliert, die die australische Tierwelt von derjenigen Asiens abtrennte..$^{43}$ Insbesondere Fritz Sarasin begeisterte sich für die Tiergeographie, die er auf eine kritisch-anspruchsvolle Art in Auseinandersetzung mit älteren Forschern wie Wallace beinahe more philologico betrieb, sowohl mit der Frage nach den geologisch-historischen Beziehungen zwischen Sri Lanka / Ceylon und Südindien, als auch mit For-

41 Zur Entwicklung der biologischen (im modernen Wortsinn) Forschung in der zweiten Hälfte des 19. Jahrhunderts Nyhart 1995; Jardine 2005. Zu den Konzepten «Labscape vs. Landscape» Kohler 2002. Zum Verhältnis zwischen Sammeln und Experimentieren in der Praxis der Naturgeschichte zuletzt Strasser 2011; früher Latour 1995 (Hinweis bei Vinck 2010, 207f.).

42 Wir fassen hier die Ergebnisse aus der Analyse der Briefe der beiden Sarasin an Ludwig Rütimeyer (Staatsarchiv Basel-Stadt, Privatarchiv PA 212a, T2 I Band I 1884-1895, 31 Briefe der Sarasin an Rütimeyer) und den Ratsherrn Dr. Fritz Müller (1834-1895) (ebenda, 54 Briefe) zusammen. Zu Fritz (Friedrich) Müller (1834-1895): Lotz/Rütimeyer 1896.

43 Raby 2002; Browne 1983; Junker 2000, 363f., 380. Wallace war für die Autoren des 19. Jahrhunderts ein äußerst wichtiger Ausgangspunkt, so für Semper 1880, 2. Teil, 137ff., für Ludwig Rütimeyer und die beiden Sarasin. Zur aktuellen Biogeographie der Region, in welcher die «Wallace Line» verläuft, vgl. http://www.wallacea.info/biogeography, besucht am 22.6.2012. 
schungen zu Sulawesi / Celebes und schließlich mit einer Exkursion nach Neukaledonien und den benachbarten Loyalty-Inseln. ${ }^{44}$

Paul und Fritz Sarasin waren somit Vertreter einer eigentlichen NaturGeschichte, aufbauend auf den von Rütimeyer vermittelten Traditionen und der damaligen Darwin-Rezeption, verankert in der Wissenschaftsauffassung der 1880er Jahre («reine Wissenschaft» mit ihrem eigenen Ethos der Wahrheitssuche) und den Methoden der Suche nach «Dokumenten» der Naturgeschichte in mehreren, komplementären Richtungen (vergleichende Anatomie, Paläontologie, Geologie, Embryologie, Tier- und Pflanzengeographie), die sie in Würzburg angetroffen und aufgegriffen hatten. Während Naturgeschichte die erwähnte Suche nach den Verwandtschaften der Arten in den Dimensionen der Zeit und des Raums bedeutete, hatte der Entwicklungsbegriff eine Schlüsselfunktion als ordnende Vorstellung. Verbunden wurden dabei die Forschungsorte des Feldes (Exkursion, Forschungsreise), des Labors (in Berlin, später in Basel) und der Sammlung (naturhistorisches Museum). Verkörpert war diese Verbindung in der Existenz der GentlemanForschungsreisenden, die zwischen den Exkursionen in einer Hauptstadt des Wissens (zuerst Berlin, später Basel) residierten, vor allem Inventare ihrer wissenschaftlich durch sie selbst, aber teilweise durch beauftragte Fachleute bestimmten Funde publizierten und sich schließlich, einmal etwas angegraut, im Basler Museum einrichteten. Dies galt jedenfalls für Fritz, der seine Stellung in den wissenschaftlichen Sammlungen in Basel mit Überzeugung und Ausdauer festigte, während Paul nach einer Phase des Interesses für die Ur- und Frühgeschichte zunehmend spekulativeren Forschungen nachging.

\section{Naturschutz national und international}

Eine Verbindung von Naturgeschichte und Naturschutz scheint zunächst ganz unwahrscheinlich zu sein. Naturgeschichtler erforschten die Natur dadurch, dass sie sie zerstörten, um sie in Bruchstücken ins Labor und dann ins Museum (ein «Reich toter Tiere $»^{45}$ ) mitzunehmen. Auf ihren Exkursionen in Südländer wurde richtig abgeräumt: Hunderte von Tieren einer Art oder

44 Exemplarisch sind zum Entwicklungsgedanken die gemeinsam publizierten Studien aus Sri Lanka: Sarasin, F. und P.1887-1893; dann die Arbeiten von Fritz Sarasin zur Tiergeschichte und Tiergeographie, darunter: Sarasin, F. 1909, Sarasin, F. 1910, Sarasin, F. 1925. Ferner Sarasin, F., Manuskript eines Vortrags im Archiv des Museums der Kulturen Basel unter dem Titel Über die Tiergeschichte der Länder des südwestlichen Pazifischen Ozeans, 42 durchpaginierte Seiten Handschrift, mit Hinweisen auf Lichtbilder, undatiert.

45 Hafner 2011 zeigt unter dieser Überschrift ein Bild der Galerie d'Anatomie Comparée des Muséum National d'Histoire Naturelle (Paris). 
Familie wurden gejagt, erlegt, provisorisch konserviert, in Gläser verpackt, in Konservendosen eingelötet und per Schiff und Bahn nach Europa verfrachtet. Vieles verdarb unterwegs oder ging verloren. Was ankam und nicht verrottet war, wurde bestimmt; erst dabei erwies sich, was wirklich interessant und wissenschaftlich wertvoll war, wie bisher unbekannte Arten - und das war jeweils ein Bruchteil des Materials. ${ }^{46}$

Gerade solche Arten, von denen man bereits wusste, dass sie selten oder im Aussterben begriffen waren, wurden als Trophäen der Wissenschaft in die Museen in Europa eingebracht, oft nur als Balg, manchmal als Skelett. Wünschbar war, vom selben Exemplar beides zu erhalten. ${ }^{47}$ Die Sarasin selbst töteten in Ceylon Elefantenkühe auf den bloßen Verdacht hin, sie könnten trächtig sein und Embryonen für die Untersuchung liefern. ${ }^{48}$ Ein verwaistes Elefantenkalb ließen sie dann nach Basel verfrachten, wo es lebend ankam und ab 1886 im Zoo zur Schau gestellt wurde, man nannte es «Kumbuk». Als diese Elefantenkuh nach ihrem Tod präpariert und im Museum ausgestellt werden sollte, zersetzte sich der riesige Balg jedoch während der langen Wartezeit auf seine Verwendung in einem Bassin im Museumshof. ${ }^{49}$

Die Medaille der durchaus kolonialistischen Ausräumung und Aneignung der Tropen durch Europäer hatte aber auch eine andere Seite. Nicht nur die toten Objekte im Museum dokumentierten «Entwicklung» durch die Bezie-

46 Einen Eindruck von den Verlusten gibt die Korrespondenz zwischen den Vettern Sarasin und den Basler Museumsverantwortlichen Rütimeyer und Müller während der Kampagnen in Sri Lanka (ähnlich später in Sulawesi), StABS PA 212 a Sarasinsches Familienarchiv, T2. - Sarasin, Ph. 2009, 24, 33 ist das verlustreiche «Abräumen» anhand der Literatur über Darwin und die britischen Museumssammlungen ebenfalls aufgefallen.

47 Die Korrespondenzen der Zoologischen Abteilung des Basler naturhistorischen Museums (faktisch die an Fritz Sarasin und Jean Roux gerichteten Briefe) enthalten zahlreiche Beispiele für die Verfolgung dieser Ziele. Naturhistorisches Museum Basel, Archiv.

48 Foto von der Elefantenjagd mit den üblichen Großwildjägerposen im Museum der Kulturen, Archiv 098 H Sarasin (alte Signatur H Sar 3). Sarasin, F. 1939, 97 erzählt den Vorgang.

49 Die Geschichte von Kumbuk lässt sich in den Briefwechseln zwischen den Vettern Sarasin einserseits, Ludwig Rütimeyer und Fritz Müller andererseits nachverfolgen. Das Tier wurde als Säugling am 9.6.1885 auf Sri Lanka von den Sarasin gefangen und 1886 als Geschenk für den Zoologischen Garten nach Basel geschickt. 1891 wurde für Kumbuk - anscheinend auf Kosten der Sarasin - ein Elefantenhaus im Zoologischen Garten erbaut (Staatsarchiv Basel-Stadt, Privatarchiv 212a T2 I Briefe der Sarasin an Fritz Müller 16.10.1885, 4.1.1886, 2.5.1886, 6.7.1891). Die Schlachtung des 1917 im Zoologischen Garten Basel verstorbenen Tiers und die versuchte Präparation sind dokumentiert in der Korrespondenz von Fritz Sarasin und Jean Roux 1917 im Naturhistorischen Museum Basel (Akten zur Präparation und Konservierung der Elefantenhaut [Kumbuk], ebenda Schreiben des Präparators Gustav Schneider Basel an Dr. Fritz Sarasin vom 15.9.1921), in den Protokollen der Naturhistorischen Kommission (Naturhistorisches Museum Basel, Archiv, Protokolle der naturhistorischen Kommission des Museums in Basel, 1917-1924, Eintrag 17.1.1918) und in den gedruckten Jahresberichten des naturhistorischen Museums Basel, 1917. 
hungen der Ordnung, die die Museumsforschung zwischen ihnen herstellte. Diese toten «Buchstaben im Buch der Naturgeschichte» mussten durch die lebendigen Zeichen der Entwicklung ergänzt werden. Lebewesen in ihrer angestammten Umwelt, die man sich als möglichst unberührt erhoffte, waren ebenso wichtige «Dokumente» wie deren tote Überreste. Der Vorteil dabei war, dass sie in Reproduktion und Interaktion miteinander und mit ihrer Umwelt, also «biologisch», beobachtet werden konnten und künftiger Forschung zugänglich blieben.

Die Hauptdimensionen der Entwicklung, Ort, Zeit und Verwandtschaft, sollten nach Ansicht der Sarasin in Gestalt lebendiger Anschauung verfügbar sein. Auch das war im Grunde ein kolonialistischer Zug; denn Menschen, Tiere und Pflanzen der Tropen wurden vor allem in ihrer Eigenschaft als Entwicklungsdokumente geschätzt und weniger für das, was sie in ihrer angestammten Region und in ihrem eigenen Kontext bedeuteten. Nicht Kommunikation, sondern Dokumentation war das Ziel, wie sich insbesondere beim Umgang mit Menschen zeigt.

«Ort» stand für die Kontexte: Bemerkenswerte Variationen der Spezies Homo sapiens sollten im Zusammenhang mit der Natur, die ihnen Nahrung lieferte, mit der Landschaft, die ihr Lebensraum war, mit den Gerätschaften für Krieg, Ackerbau, Jagd, Transport, Kult erhalten bleiben; Ähnliches galt für interessante Tiere im Kontext ihrer Nahrung und ihrer Prädatoren.

«Zeit» stand für Unberührtheit - denn eine direkte, unvermischte Herkunft aus einer Urzeit war entscheidend für die Wertschätzung. Für die Zukunft sollten sie in diesem ursprünglichen Zustand bewahrt und darum geschützt werden. Wo die Unberührtheit verlorengegangen war, durch Kulturkontakt und Kommerz mit den Weißen, durch landschaftliche Veränderungen im Zusammenhang mit dem Abbau von Bodenschätzen, durch Bejagung zum Erzielen von kapitalistischem Gewinn, sollte die Isolierung im Reservat den Organismen Zeit geben für eine Zurückentwicklung in die Zustände der Ursprünglichkeit. ${ }^{50}$

Eine Verlusterfahrung prägte die Generation, die ganz unmittelbar die Effekte der Dampfschifffahrt nach Fahrplan, der Erschließung mit der Eisenbahn, der Befriedung durch modern ausgerüstete Truppen, der Missionierung durch viele Konfessionen, des Anschlusses immer neuer Landstriche und Uferstrecken an die kapitalistische Weltwirtschaft unter den Bedingungen des Maschinenzeitalters erleben und beobachten konnte. Zwar halfen alle diese Errungenschaften, das Unberührte, Ursprüngliche überhaupt erreichbar zu machen, die Natur zu finden, sich anzueignen und sie mitzu-

50 Sarasin, P. 1910. Zu Fritz Sarasin über Neukaledonien siehe unten Anm. 57.

Gesnerus 71 (2014) 
nehmen. Zugleich aber zerstörten diese Errungenschaften der Moderne das Studienobjekt schrittweise, nicht in Jahrhunderten oder Jahrzehnten, sondern innerhalb weniger Jahre.

Für die Sarasin war Ludwig Rütimeyer die Referenzfigur für dieses Empfinden. Zwar war er selbst nicht über Sizilien hinausgekommen und hatte das meiste Wissen über ferne Länder aus den Museumsbesuchen in London, in Paris und in vielen weiteren europäischen Sammlungen sowie aus der umfassend verarbeiteten Forschungsliteratur erworben. Er hatte aber schon lange vor den Sarasin formuliert, dass der «weiße Mann» alles zerstöre, was er antreffe.

Überall, wo die kaukasische Rasse auf solchem Kampfplatz [eine Anspielung auf den «Kampf ums Dasein»] auftritt, kann ein für Tiergeschichte aufmerksames Ohr den Ruf vernehmen: Ave Caesar, morituri te salutant. ${ }^{51}$

Diese aus einer konservativen, antimodernistischen Kulturkritik heraus formulierte Anklage setzte sich bei den Sarasin ungebrochen fort. Sie erlebten den «weißen Mann» als den Störenfried im irdischen Paradies. Paul Sarasin zitierte die oben angeführte Passage aus Rütimeyer zustimmend in seinem Referat über den Weltnaturschutz von 1910. Dabei vergaß er nicht, die Verbindung zwischen Naturschutz und wissenschaftlicher Untersuchung der Entwicklung der Arten herzustellen, indem er auch den in Rütimeyers Text vorausgehenden Satz anführte:

Szenen ungestörten Tierlebens, wie sie die fromme Erinnerung bezeichnend von dem Morgenlichte der letzten Schöpfung beleuchten lässt, sind nur noch den kühnsten Reisenden zugänglich, die ins Innere ältester Schauplätze der Speziesbildung eindringen. ${ }^{52}$

Ohne jeden Zweifel verstanden sich die Sarasin als solche «kühnste Reisende», und die Verbindung der Suche nach unberührter Natur an «Schauplätzen der Speziesbildung» mit Bestrebungen des weltweiten Naturschutzes war ihr eigenes Programm geworden.

So wurde der Naturgeschichtler zum Zeitgenossen der beschleunigten Naturzerstörung und zum Anwalt der Naturbewahrung in Reservaten, in denen eine Art lebendiger Museen entstehen sollte, total geschützt, wo nötig durch die Gewalt von Waffen, frei von jeglichem Verkehr mit der Außenwelt, nur sporadisch besucht von der Wissenschaft. ${ }^{53}$

Aufschlussreich ist die Darstellung des flugunfähigen Vogels Kagu (Rhynochetus jubatus) auf Neukaledonien durch Fritz Sarasin. Dieser Vogel war zunächst im Basler Naturhistorischen Museum nur als Präparat bekannt,

51 Rütimeyer, Ludwig 1898, Band 1, 222.

52 Sarasin, P. 1910, 70, 93.

53 Kupper 2012, $83 f$. 
das ein Züchter aus Nouméa dorthin geschickt hatte. ${ }^{54}$ Sarasin erforschte den Vogel in seinem Lebensraum. Er verstand die angetroffene Situation als eine Etappe des Aussterbens der Art und analysierte die Faktoren, die zum Populationsrückgang führten: Einführung von Hunden auf der Insel durch weiße Siedler, Verwendung dieser Hunde durch «Kanaken» für die Jagd, die Reduktion des Lebensraumes durch den Nickel-Bergbau, der Fang von Kagus, die in Nouméa als Haustiere beliebt waren. Sarasins Darstellung stand im Kontext einer Kritik der französischen Kolonialherrschaft: ${ }^{55}$ Diese ließ Prozesse sich frei entfalten, die das Aussterben des Vogels beschleunigen mussten. Die Art selbst war für Fritz Sarasin ein Dokument der Evolution: eine Art ohne natürliche Feinde, die friedfertig am Boden lebte, in Büschen nistete und deren einziger Verteidigungsreflex darin bestand, dass sich der angegriffene Vogel duckte. Damit war er unter den neuen, durch die koloniale Erschließung geschaffenen Umständen wehrlos der Vernichtung ausgesetzt. Wie bereits bemerkt, regte die Feststellung, dass das Tier vom Aussterben bedroht war, jedoch erst recht dazu an, Exemplare zu töten und daraus Präparate, auch Schaustücke, für das Museum fertigen zu lassen. ${ }^{56}$

Selbstverständlich gab es daneben auch naturästhetische Begründungen. Am leichtesten zu vermitteln war die Notwendigkeit des Schutzes für charismatische Arten, den stolzen Adler, den mächtigen Seelöwen, den gewaltigen Wal, im Sinne von Brehms Naturbildern, und für Organismen, die die «herrlichste Zierde unseres Landes» (z.B. Edelweiß für die Schweiz) darstellten. Diese Natur galt es vor «Verarmung» zu bewahren, eine «verödete Welt» zu verhindern. ${ }^{57}$ Die Sarasin waren selbst Kapitalistenkinder und -enkel, die zur weltbereisenden und forschenden internationalen «Gentry» gehörten. Dennoch entwickelten sie gegen das, was sie nun selbst den «Kapitalismus» nannten, eine starke Abneigung. Nach ihrer Auffassung verwandelte dieser die Schönheit der Welt in das Hässliche von Maschinen und kurzfristigem Profit. Der von ihnen angeprangerte «Kapitalismus» erkannte in der Welt und in den Naturdingen nur Ressourcen, die als Geldwert und als Quellen spekulativen Gewinns geschätzt wurden. ${ }^{58}$ Ursprüngliche,

54 (Gedruckter) Bericht über das Naturhistorische Museum per 1899, 270: Benjamin Amstein aus Nouméa schenkt dem Museum einen Nestling und ein Ei von Rhy(i)nochetus jubatus. Dasselbe 1913: Fritz Sarasin und Jean Roux schenken dem Museum ein Skelett des Vogels.

55 Kritik an der französischen Untätigkeit im Naturschutz auch bei Sarasin, P. 1914, 57.

56 Sarasin, F. 1913, 55-60 mit Tafel III Fig. 17 und 18. Sarasin, F., Vortragsmanuskript Kaledonien/Vortrag französisch (Museum der Kulturen, Archiv); darin empfiehlt er den Mont Humboldt als Reservat für den Kagu (Cagou).

57 Die Hinweise auf charismatische Arten häufen sich bei Sarasin, P. 1914, 28, 31, 42 etc.

58 Sarasin, P. 1914, 58 definiert den Zweck der Totalreservate als die Erhaltung «ursprünglicher Gesamtnatur», und 74 mit Einschluss der dort lebenden Naturvölker. Ähnliche Sätze stehen in: Sarasin, P. 1910, 51, 53. 
vom «Kapitalismus» (noch) unberührte Natur wurde als harmonisch und friedlich wahrgenommen; die «in Verborgenheit dahinlebenden» und dadurch zunächst noch geschützten «Geschöpfe» führten nach Paul Sarasin eine «freudige Existenz». ${ }^{59}$

Das Grundmuster, das für die forschenden Naturschützer konstitutiv war, ließ sich jedoch weder auf eine bestimmte Ästhetik noch auf einen neukonservativen Antikapitalismus reduzieren. Es war vielmehr die Deutung der Welt, der Natur und des naturnahen, «ursprünglichen» Menschen als «Quelle» der Naturgeschichte, die der Argumentation ihren Zusammenhang verlieh.

Wie wir gesehen haben, wurde «Quelle» im naturhistorischen Sinne verstanden: ein Dokument voller Informationen für die Wissenschaftler, die gelernt hatten, dieses Dokument zu «lesen». Die schönste Aufgabe des Naturschutzes war nach Paul Sarasin die Erhaltung der «primitiven Völker», die möglichst unversehrt für die Studien unserer Nachfahren erhalten bleiben sollten. Es war für ihn ein glücklicher Zufall, dass Varietäten von Homo sapiens, deren Lebens- und Denkweise ein Entwicklungsstadium unserer eigenen Kultur repräsentierten und deren Körper eine frühere Erscheinungsform unserer physischen Existenz erkennen ließen, erhalten geblieben waren. Diese zu untersuchen bedeutete für ihn, wie von oben herab einen Blick auf unsere eigene Vergangenheit zu werfen. Es gäbe keinen Zweifel darüber, dass deren Erhaltung eine Aufgabe des Weltnaturschutzes sei. ${ }^{60}$ Mensch und Tier seien «Blutsverwandte», und dies gelte nicht nur in physischer Hinsicht, sondern auch in psychischer. ${ }^{61}$ «Quellen» galten als unwiederbringlich. Eine bestimmte «Quelle» konnte die einzige Spur sein, die die Erkenntnis des Zusammenhangs, der Entwicklung gestattete, und so müssten die lebendigen Dinge in der Natur wenigstens exemplarisch in bewachten Reservaten ebenso gut geschützt werden, wie deren tote Zeugnisse in den Schränken, Gläsern, Schubladen und Kisten der Museen dem zerstörerischen Zugriff des Unwissenden, sensations- und profitgierigen modernen Menschen entzogen und für die Zwecke der reinen Wissenschaft bewahrt wurden.

Naturschutz wurde noch vor dem Ersten Weltkrieg international. Dies ergab sich nicht nur daraus, dass die Natur keine Grenzen kennt und deshalb übernational durch Konventionen geschützt werden muss, sondern auch aus

59 Sarasin, P. 1910, 51.

60 Sarasin, P. 1910, 51ff. referiert die ersten Jahre der Geschichte des Naturschutzes in der Schweiz und die Initiative zum Weltnaturschutz bis 1910. Ausgangspunkt war die Gründung der Schweizerischen Naturschutzkommission am 1.8.1906 aus der Schweizerischen Naturforschenden Gesellschaft heraus. Kupper 2012, 82ff.

61 Sarasin, P. 1914, 74-80. Zum «anthropologischen Naturschutz» Wöbse 2006 und Wöbse 2012, $36 \mathrm{ff}$. 
der Perspektive der globalisierten Zerstörung durch den entfesselten Kapitalismus, die der weltreisende Naturforscher aus eigener Anschauung kannte. Erleichtert wurde ein solches Projekt vor dem Ersten Weltkrieg durch die internationalen Kontakte der Mächtigen, der Reichen und der Gelehrten eine Kommunikationskultur, die 1914-1918 zerstört wurde.

So wurden die Sarasin vor 1914 zu Pionieren des Naturschutzes in der Schweiz, ${ }^{62}$ zu Kritikern der Habitatzerstörung in den Kolonialgebieten und zu Propagandisten der Idee des «totalen Reservats». Dies implizierte auch den ambivalenten «Menschenschutz», der einerseits die Hinwendung zu den unter der Kolonisierung leidenden Teilen der einen Menschheit bedeutete, andererseits in einer Art von gezielter Absonderung diesen Menschen bestimmte Territorien und bestimmte Lebensformen zuwies und ihnen damit eine Teilhabe an der weiteren Entwicklung der Welt verweigerte. Während das totale Reservat in den Kolonien vorerst utopisch blieb, gelang es den Vettern Sarasin, mit dem schweizerischen Nationalpark ein Modell für ein Totalreservat in der Nordhemisphäre zu lancieren - wenn auch unter Ausschluss von Menschen. Paul Sarasin erreichte 1906 die Schaffung einer Kommission für den Naturschutz in der Schweiz, 1908 entwickelte er den Plan für den Nationalpark und 1909 gründete er den Naturschutzbund zur Finanzierung dieses Parks. 1910 plädierte er am Zoologenkongress in Graz für die erwähnte Idee eines Weltnaturschutzes; diese Forderung wiederholte er an der Zusammenkunft der Naturforschenden Gesellschaft in Basel desselben Jahres. 1913 fand zwar noch die damals beschlossene internationale Konferenz in Bern statt, aber die auf den Herbst 1914 geplante Gründung des Weltnaturschutzes in Basel wurde nach dem Kriegsausbruch vom August 1914 unmöglich.

In den Reservaten sollte geforscht werden und nicht - wie in den amerikanischen Nationalparks - ein Erholungs-, Bildungs- oder Sensationsbedürfnis befriedigt werden; ${ }^{63}$ auch wurden kommerzielle Aktionen zur Finanzierung des Schutzes aus dem Sarasin'schen Konzept ausgeschlossen. Das Reservat diente nur der Wissenschaft - aber nicht sofort, es sollte sich zuerst erholen, um durch das Walten des Faktors Zeit seine Ursprünglichkeit zurückzugewinnen. Eine Forschungskommission für den Nationalpark wurde erst 1916 gebildet. ${ }^{64}$

62 Burckhardt 1992; Bachmann 1999; Kupper 2012.

63 Kupper 2009; Gissibl 2012.

64 Staatsarchiv Basel-Stadt Privatarchiv PA 924 Schweizer Bund für Naturschutz, M 3.2 Teil 1 Nationalpark. Eidg. Nationalparkkommission, Protokolle 1914-1929. Im Protokoll für 8.-14. Juli 1918 wird vermerkt, dass die Präsenz von 21 Forschern im Nationalpark während eines einzigen Sommers eine sehr große Belastung darstelle. Zu dieser Bemerkung auch Kupper 2012, 205. Das Forschungsprogramm bei Kupper 2012, 184ff. 


\section{Ergebnisse}

Der 1914 gegründete schweizerische Nationalpark hatte seine Ursprünge in Sri Lanka, Sulawesi und Neukaledonien. Er war eine Reaktion auf die Auseinandersetzung mit dem Versagen von Kolonialbehören und mit den Ursachen der ungebremsten, mechanisierten («kapitalistischen») Ausbeutung der Weltmeere, der tropischen und arktischen Inseln zu Zwecken des raschen Profits. Er war eine Folge der globalen Verlusterfahrung von Forschungsreisenden, die einer naturgeschichtlichen Entwicklungskonzeption anhingen.

Das Schutzkonzept war nicht nur aus der Ästhetik der erhabenen Natur, der Bewunderung für charismatische Geschöpfe, hergeleitet, sondern es folgte mehr noch aus der Ansicht, dass die Natur ein Inventar von «Dokumenten» der Entwicklungsgeschichte sei. Jedes Exemplar, das verlorenging, konnte den Verlust einer Information bedeuten, die für die Rekonstruktion der Verwandtschaften zwischen den Arten, für die Erkenntnis des Verlaufs der Entwicklungsgeschichte in den geologischen Zeiträumen und für die Erforschung der Geschichte der geographischen Verbreitung von Arten über den Globus eine Schlüsselfunktion haben könnte. Die Inventarisierung und Erschließung toter Zeugnisse des Lebens in den geordneten Depots der Museen sollte durch geschützte und damit unberührte Orte in Reservaten ergänzt werden, in denen Leben in seiner Ursprünglichkeit zu bewahren sei, damit es für die künftige Forschung erhalten bliebe.

\section{Bibliographie}

Adams, Mark B. (ed.), The Evolution of Theodosius Dobzhansky. Essays on his Life and Thought in Russia and America (Princeton, N.J. 1994)

Arnst,Andreas/Walter Jaeschke (Hg.), Materialismus und Spiritualismus. Philosophie und Wissenschaften nach 1848 (Hamburg 2000)

Bachmann, Stefan, Zwischen Patriotismus und Wissenschaft. Die schweizerischen Naturschutzpioniere (1900-1938) (Zürich 1999)

Baer, Karl Ernst von, Über Entwickelungsgeschichte der Thiere. Beobachtung und Reflexion, 2 Bände (Königsberg 1828/1837)

Balmer, Heinz, «Rütimeyer, Ludwig», Historisches Lexikon der Schweiz, http://www. hls-dhs-dss.ch/textes/d/D28925.php, besucht 1.7.2012

Bayertz, Kurt/Walter Jaeschke/Myriam Gerhard (Hg.), Weltanschauung, Philosophie und Naturwissenschaft im 19. Jahrhundert. Der Materialismusstreit. Band 1 (Hamburg 2007)

Bouyssi, François, Alfred Giard (1846-1908) et ses elèves, un cénacle de "philosophes biologistes» aux origines du scientisme? (Lille 2001) 
Bowler, Peter J., Evolution. The History of an Idea (Berkeley 2003)

Browne, Janet, The Secular Ark. Studies in the History of Biogeography (New Haven/ London 1983)

Burckhardt, Dieter, «Die Wiege des Naturschutzes stand in Basel. Streiflichter auf Entstehung und Entwicklung des Naturschutzes in der Schweiz», Verhandlungen der Naturforschenden Gesellschaft in Basel 102 (1992) 3-45

Burckhardt, Lukas u.a. (Hg.), Das politische System Basel-Stadt. Geschichte, Strukturen, Institutionen, Politikbereiche (Basel/Frankfurt/Main 1984)

Cairns, John/Gunther S. Stent/James D. Watson (eds.), Phage and the Origins of Molecular Biology (Cold Spring Harbor NY 1992)

Carlson, Elof Axel, Genes, Radiation, and Society. The Life and Work of H. J. Muller (Ithaca N.Y 1981)

Chadarevian, Soraya de, "Portrait of a Discovery. Watson, Crick, and the Double Helix", Isis 94 (2003) 90-105

Darwin, Charles, The Descent of Man, and Selection in Relation to Sex (London 1871)

Darwin Correspondence Database, http://www.darwinproject.ac.uk/namedef-3905, besucht 11.6.2012

Fischer, Ernst Peter/Carol Lipson, Thinking about Science. Max Delbruck and the Origins of Molecular Biology (New York 1988)

Gedächtnis-Ausstellung Fritz und Paul Sarasin, Sonderausstellung in Zusammenarbeit mit dem Naturhistorischen Museum Basel vom 1. Juli bis 1. November 1959 [im Museum für Völkerkunde Basel] (Basel 1959)

Geus, Armin, «Zoologische Disziplinen», in: Ilse Jahn (Hg.), Geschichte der Biologie. Theorien, Methoden, Institutionen, Kurzbiographien (Heidelberg/Berlin 2000) 324-355

Gissibl, Bernhard/Sabine Höhler/Patrick Kupper (eds.), Civilizing Nature. National Parks in Global Historical Perspective (New York/Oxford 2012)

Gliboff, Sander, "H. G. Bronn and the History of Nature", Journal of the History of Biology 40 (2007) 259-294

Gliboff, Sander, H. G. Bronn, Ernst Haeckel, and the Origins of German Darwinism. A Study in Translation and Transformation (Cambridge MA 2008)

Green, Christopher D., "Scientific Objectivity and E. B. Titchener's Experimental Psychology", Isis 101 (2010) 697-721

Gregory, Fredrick, Scientific Materialism in Nineteenth Century Germany (Berlin 1977)

Gümbel, Wilhelm von, «Bronn, Heinrich Georg», Allgemeine Deutsche Biographie 3 (1876) 355-360, Digitale Volltext-Ausgabe in Wikisource, http://de.wikisource. org/w/index.php?title=ADB:Bronn,_Heinrich_Georg\&oldid=1695233, besucht 15.6.2012

Haeckel, Ernst, Generelle Morphologie der Organismen. Allgemeine Grundzüge der organischen Formen-Wissenschaft, mechanisch begründet durch die von Charles Darwin reformierte Descendenz-Theorie (Berlin 1866)

Hafner, Urs, «Im Reich der toten Tiere», Schweizerischer Nationalfonds - Horizonte (Dezember 2011) 35

His, Euard, Basler Gelehrte des 19. Jahrhunderts (Basel 1941)

Jahn, Ilse (Hg.), Geschichte der Biologie. Theorien, Methoden, Institutionen, Kurzbiographien (3. Aufl., Berlin 2000) 
Janner, Sara, Zwischen Machtanspruch und Autoritätsverlust. Zur Funktion von Religion und Kirchlichkeit in Politik und Selbstverständnis des konservativen alten Bürgertums im Basel des 19. Jahrhunderts (Basel 2012)

Jardine, Nicholas u.a. (eds.), Cultures of Natural History (Cambridge 2005)

Junker, Thomas, «Charles Darwin und die Evolutionstheorien des 19. Jahrhunderts», Ilse Jahn (Hg.), Geschichte der Biologie. Theorien, Methoden, Institutionen, Kurzbiographien (3. Aufl., Berlin 2000) 356-385

Kohler, Robert E., Lords of the Fly. 'Drosophila' Genetics and the Experimental Life (Chicago 1994)

Kohler, Robert E., Landscapes and Labscapes. Exploring the Lab-Field Border in Biology (Chicago 2002)

Kohler, Robert E., All Creatures. Naturalists, Collectors, and Biodiversity, 1850-1950 (Princeton 2006)

Kupper, Patrick, "Science and the National Parks. A Transatlantic Perspective on the Interwar Years", Environmental History 14 (2009) 58-81

Kupper, Patrick, Wildnis schaffen. Eine transnationale Geschichte des Schweizerischen Nationalparks (Bern 2012)

Latour, Bruno, "The Pedofil of Boa Vista. A Photo-Philosophical Montage", Common Knowledge 4 (1995) 144-187

Lenoir, Timothy, "Essay Review: The Darwin Industry", Journal of the History of Biology 20 (1987) 115-130

Lienhard, Luc, «Christ, Hermann», Historisches Lexikon der Schweiz, www.hls-dhsdss.ch/textes/d/D28801.php, besucht 1.7.2012

Loison, Laurent, Qu'est-ce que le néolamarckisme? Les biologistes français et la question de l'évolution des espèces, 1870-1940 (Paris 2010)

Lotz, Theodor/Leopold Rütimeyer, «Dr. Friedr. Müller geboren 1834 gestorben 10. März 1895», Sonderabdruck aus: Verhandlungen der Naturforschenden Gesellschaft in Basel 11 (1896) 1-25

Lyotard, Jean-François, La condition postmoderne (Paris 1979)

Mayr, Ernst, "Semper, Karl”, Dictionary of Scientific Biography 12 (1975) 299

Mayr, Ernst, Systematics and the Origin of Species from a Viewpoint of a Zoologist (Cambridge MA 1999, original edition 1942)

Mayr, Ernst, The Growth of Biological Thought. Diversity, Evolution, and Inheritance (Cambridge MA 1982)

Mayr, Ernst/William B. Provine (eds.), The Evolutionary Synthesis. Perpectives on the Unification of Biology (Cambridge MA/London 1980)

Meyer von Knonau, Gerold, «Rütimeyer, Karl Ludwig», Allgemeine Deutsche Biographie 53 (Leipzig 1907) 654-657

Nyhart, Lynn K., Biology Takes Form. Animal Morphology and the German Universities, 1800-1900 (Chicago 1995)

Portmann, Adolf, «Die Frühzeit des Darwinismus im Werk Ludwig Rütimeyers», Basler Stadtbuch 85 (1965) 164-188

Raby, Peter, Alfred Russel Wallace. A Life (Princeton 2002)

Recueil des procès-verbaux de la Conférence internationale pour la protection de la nature (Berne 1913)

Richards, Robert J., "The Moral Grammar of Narratives in History of Biology. The Case of Haeckel and Nazi Biology", in: Michael Ruse/David Hull (eds.), Cam- 
bridge Companion to the Philosophy of Biology (Cambridge 2007): http://home. uchicago.edu/ rjr6/articles/Moral\%20Grammar\%20of\%20Narratives-illustrated.pdf, besucht 30.6.2012

Richards Robert J., The Tragic Sense of Life. Ernst Haeckel and the Struggle over Evolutionary Thought (Chicago/London 2008)

Riha, Ortrun/Thomas Schmuck, 'Das allgemeinste Gesetz'. Karl Ernst von Baer (17921876) und die grossen Diskurse des 19. Jahrhunderts (Aachen 2011)

Rütimeyer, Leopold, «Paul Sarasin», Basler Jahrbuch 51 (1931) 195-209

Rütimeyer, Ludwig, Über die Aufgaben der Naturgeschichte. Vortrag bei der Jahresfeier der Universität Basel im November 1865 (Basel 1865)

Rütimeyer, Ludwig, «Besprechung von Ernst Haeckel, 'Über die Entstehung und den Stammbaum des Menschengeschlechts' und 'Natürliche Schöpfungsgeschichte'», Archiv für Anthropologie 3 (1868) 301-302

Rütimeyer, Ludwig, Die Grenzen der Thierwelt. Eine Betrachtung zu Darwin's Lehre. Zwei in Basel gehaltene Vorträge (Basel 1868)

Rütimeyer, Ludwig, Die Veränderungen der Tierwelt in der Schweiz seit Anwesenheit des Menschen (Basel 1875)

Rütimeyer, Ludwig, Über die Art des Fortschritts in den organischen Geschöpfen. Eröffnungsrede bei der 59. Jahresversammlung (21. August 1876) der schweizerischen naturforschenden Gesellschaft in Basel (Basel/Genf 1876)

Rütimeyer, Ludwig, «Ungeordnete Rückblicke auf den der Wissenschaft gewidmeten Teil meines Lebens, geschrieben in den Jahren 1888-1895», Ders., Gesammelte kleine Schriften allgemeinen Inhalts aus dem Gebiete der Naturwissenschaft nebst einer autobiographischen Skizze, hg. v. Hans Georg Stehlin, Bd. 1, Autobiographie [und] zoologische Schriften (Basel 1898) 1-39

Rütimeyer, Ludwig, «Charles Darwin», Ders., Gesammelte kleine Schriften allgemeinen Inhalts aus dem Gebiete der Naturwissenschaft nebst einer autobiographischen Skizze, hg. v. Hans Georg Stehlin, Bd. 2, Geographische Schriften, Necrologe, Verzeichnis der Publicationen (Basel 1898) 371-386

Rütimeyer,Ludwig, Briefe von Ludwig Rütimeyer 1825-1895 als Manuskript gedruckt (Basel 1902)

Sarasin, Fritz, «Über die Geschichte der Tierwelt in Ceylon», Verhandlungen der Schweizerischen Naturforschenden Gesellschaft, 92. Jahresversammlung vom 5.-8. September 1909 in Lausanne, Bd. 1 Vorträge und Sitzungsprotokolle (Aarau) $117-138$

Sarasin, Fritz, «Über die Geschichte der Tierwelt von Ceylon», Zoologische Jahrbücher, Supplement 12, Heft 1 (Jena 1910)

Sarasin, Fritz, «Die Vögel Neu-Caledoniens und der Loyalty-Inseln», Fritz Sarasin und Jean Roux (Hg.), Nova Caledonia. Forschungen in Neu-Caledonien und auf den Loyalty-Inseln, Zoologie Bd. 1 Teil 1 (Wiesbaden 1913) 1-78

Sarasin, Fritz, «Über die Tiergeschichte der Länder des Südwestlichen Pazifischen Ozeans auf Grund von Forschungen in Neu-Caledonien und auf den LoyaltyInseln», Fritz Sarasin und Jean Roux, Nova Caledonia. Forschungen in NeuCaledonien und auf den Loyalty-Inseln, Teil A. Zoologie Vol. IV, L. 1 (Berlin 1925) 1-177

Sarasin, Fritz, «Zur Erinnerung an Paul Benedikt Sarasin 1856-1929», Verhandlungen der Naturforschenden Gesellschaft in Basel 40 (1928/29) 1-28 
Sarasin, Fritz, Reisen und Forschung in Ceylon in den Jahren 1883-1886, 1890, 1901, 1907 und 1925 (Basel 1939)

Sarasin, Fritz, «Geschichte der Vogelsammlung des Basler Naturhistorischen Museums», Verhandlungen der Naturforschenden Gesellschaft in Basel 51 (1939) 13-24

Sarasin, Fritz, Aus einem glücklichen Leben. Biographische Notizen (Basel 1941)

Sarasin, Fritz, Kaledonien - Vortrag französisch. Museum der Kulturen Basel, Archiv 09 9H Sarasin, undatiertes Vortragsmanuskript (Société de Géographie Paris)

Sarasin, Fritz, Über die Tiergeschichte des südwestlichen Pazifischen Ozeans. Museum der Kulturen Basel, Archiv 09 9H Sarasin, undatiertes Vortragsmanuskript

Sarasin, Fritz und Paul, Korrespondenz, Staatsarchiv Basel-Stadt, Privatarchiv PA 212a T2

Sarasin, Fritz und Paul, Ergebnisse naturwissenschaftlicher Forschungen auf Ceylon (Wiesbaden 1887-1893)

Sarasin, Fritz/Jean Roux, Korrespondenz Drs. F[ritz] Sarasin J[ean] Roux (Naturhistorisches Museum Basel, Zoologische Abteilung, Archiv)

Sarasin, Paul, Gedichte (Wiesbaden 1893)

Sarasin, Paul, Kurze Worte der Erinnerung an Ludwig Rütimeyer, gesprochen bei Gelegenheit der Enthüllung seiner Büste am 10. November 1899 (Basel 1900)

Sarasin, Paul, Ein Blütenzweig aus dem Reiche der Ideen. Gedanken und Gedichte (Basel 1908)

Sarasin, Paul, «Weltnaturschutz. Vortrag gehalten am VIII. internationalen Zoologenkongress in Graz am 16. August und an der 93. Versammlung der Schweizerischen Naturforschenden Gesellschaft in Basel am 5. September 1910», Verhandlungen der Schweizerischen Naturforschenden Gesellschaft, 93. Jahresversammlung vom 4. bis 7. September 1910 in Basel, Bd. 1 Vorträge und Sitzungsprotokolle (Aarau 1910) 50-73

Sarasin, Paul, «Exposé introductif», Recueil des procès-verbaux de la Conférence internationale pour la protection de la nature, Berne 17-19 novembre 1913 (Bern 1914) 23-82

Sarasin, Philipp, Darwin und Foucault. Genealogie und Geschichte im Zeitalter der Biologie (Frankfurt/Main 2009)

Schmitt, Michael, «Abstammung», Philipp Sarasin und Marianne Sommer (Hg.), Evolution. Ein interdisziplinäres Handbuch (Stuttgart 2010) 3-5

Semper, Carl, Reisen im Archipel der Philippinen (Wiesbaden 1867-1906)

Semper, Carl, Der Haeckelismus in der Zoologie (Hamburg 1876)

Semper, Carl, Über die Aufgaben der modernen Thiergeographie (Berlin 1879)

Semper, Carl, Die natürlichen Existenzbedingungen der Tiere (2 Teile, Leipzig 1880)

Shanahan, Timothy, The Evolution of Darwinism. Selection, Adaptation, and Progress in Evolutionary Biology (Cambridge MA/New York 2004)

Smocovitis, Vassiliki Betty, Unifying Biology. The Evolutionary Synthesis and Evolutionary Biology (Princeton 1996)

Speiser, Felix, «Dr. Fritz Sarasin, 1859-1942», Verhandlungen der Naturforschenden Gesellschaft in Basel 54 (1943) 222-264

Stehlin, Hans Georg, «Paul Sarasin (1856-1929)», Verhandlungen der Schweizerischen Naturforschenden Gesellschaft (St. Gallen/Aarau 1930) 393-407 
Strasser, Bruno, “The Experimenter's Museum: GenBank, Natural History, and the Moral Economies of Biomedicine, 1979-1982", Isis 102 (2011) 60-96

Vinck, Dominique, The Sociology of Scientific Work. The Fundamental Relationship between Science and Society (Cheltenham 2010)

Warning, Rainer (Hg.), Rezeptionsästhetik. Theorie und Praxis (München 1975)

Weingart Peter, "'Struggle for Existence'. Selection and Retention of a Metaphor", in: Sabine Maasen u.a. (eds.), Biology as Society, Society as Biology. Metaphors (Sociology of the Sciences Yearbook (Dordrecht 1994) 127-151

Weingart, Peter/ Jürgen Kroll/ Kurt Bayertz, Rasse, Blut und Gene. Geschichte der Eugenik und Rassenhygiene in Deutschland (Frankfurt a.M. 1992)

White, Hayden, Metahistory. The Historical Imagination in Nineteenth-Century Europe (Baltimore 1973)

Wiegand, F. [i.e. Paul Sarasin], Gedichte (Wiesbaden 1900)

Wöbse, Anna-Katharina, «Paul Sarasins 'antropologischer Naturschutz'. Zur 'Grösse' Mensch im frühen internationalen Naturschutz. Ein Werkstattbericht», Gert Gröning/ Joachim Wolschke-Bulmahn (Hg.), Naturschutz und Demokratie!? (München 2006) 207-214

Wöbse, Anna-Katharina, Weltnaturschutz. Umweltdiplomatie in Völkerbund und Vereinten Nationen, 1920-1950 (Frankfurt a.M. 2012)

Wuketits, Franz M., Eine kurze Kulturgeschichte der Biologie. Mythen, Darwinismus, Gentechnik (Darmstadt 1998) 